\title{
Bayesian Phylogenetic Inference via Markov Chain Monte Carlo Methods
}

\author{
Bob Mau, ${ }^{1, *}$ Michael A. Newton, ${ }^{1,2}$ and Bret Larget $^{3}$ \\ ${ }^{1}$ Department of Statistics, University of Wisconsin-Madison, \\ 1210 West Dayton Street, Madison, Wisconsin 53706-1685, U.S.A. \\ ${ }^{2}$ Department of Biostatistics and Medical Informatics, University of Wisconsin-Madison, \\ 600 Highland Avenue, Madison, Wisconsin 53792, U.S.A. \\ ${ }^{3}$ Department of Mathematics and Computer Science, Duquesne University, \\ College Hall 440, Pittsburgh, Pennsylvania 15282-1704, U.S.A. \\ *email: Robertm@genetics.wisc.edu
}

\begin{abstract}
Summary. We derive a Markov chain to sample from the posterior distribution for a phylogenetic tree given sequence information from the corresponding set of organisms, a stochastic model for these data, and a prior distribution on the space of trees. A transformation of the tree into a canonical cophenetic matrix form suggests a simple and effective proposal distribution for selecting candidate trees close to the current tree in the chain. We illustrate the algorithm with restriction site data on 9 plant species, then extend to DNA sequences from 32 species of fish. The algorithm mixes well in both examples from random starting trees, generating reproducible estimates and credible sets for the path of evolution.
\end{abstract}

KEY Words: Cophenetic matrix; Evolution; Metropolis-Hastings algorithm; Phylogeny reconstruction.

\section{Introduction}

"A phylogeny is a branching tree diagram showing the course of evolution in a group of organisms" (Felsenstein, 1983, p. 246). More and more, phylogenetic inference is based on molecular data, such as DNA or protein sequences. Given such data, life scientists wish to reconstruct the phylogeny whence these organisms arose. Their reasons are as diverse as the organisms they study. Systematists use the phylogeny to aggregate organisms into monophyletic groups, or clades, for taxonomic purposes (hence the generic term taxa). For others, the phylogeny might be of peripheral importance, yet ignoring it can lead to unwarranted conclusions. For example, given a phylogeny, comparative biologists interested in the correlation between continuous character traits in a group of organisms can correct for dependencies among species (Felsenstein, 1985b). Huelsenbeck and Rannala (1997) discuss a range of inferences that rely on the phylogeny.

Existing reconstruction techniques attempt to find the phylogeny most compatible with the data under consideration. Among the more popular methods, one can categorize those using distance matrices (e.g., Sokal and Sneath, 1963; Fitch and Margoliash, 1967) in numerical taxonomy as clustering algorithms, whereas maximum parsimony (Camin and Sokal, 1965) and maximum likelihood (Felsenstein, 1981, 1983) each optimize an objective function on the space of trees. Felsenstein (1988) provides a comprehensive review of traditional methods (see also Swofford et al., 1996).
Phylogenetic inference can also be dichotomized functionally. Maximum likelihood, maximum parsimony, and distancematrix methods are practical for data sets relating many taxa, but beyond point estimates these methods do not produce valid inferences. Measures of uncertainty rely exclusively on computer-intensive and approximate bootstrap analyses (Felsenstein, 1985a; Newton 1996). More recently developed techniques, such as the phylogenetic invariants of Cavender, Felsenstein, and Lake (see Evans and Speed, 1993; Navidi, Churchill, and von Haeseler, 1993) and a Bayesian approach (Hasegawa and Kishino, 1989; Smouse and Li, 1989; Sinsheimer, Lake, and Little, 1996), allow exact inference, but mathematical and computational complexity have constrained these methods to very small problems.

We elect a Bayesian approach and use Markov chain Monte Carlo (MCMC) methods to provide a computationally feasible technique that meets practitioners' demands for more taxa while keeping statistical inference on a sound footing, provided certain convergence criteria can be adequately demonstrated. Individual components comprising our technique can be found in the phylogenetic literature: Smouse and Li (1989) introduced the Bayesian paradigm, if not the terminology, to phylogeny reconstruction. Goldman (1993) uses nonBayesian Monte Carlo tests of significance to assess the adequacy of evolutionary models. Griffiths and Tavaré (1994a, 1994b) construct special Markov chains to compute likelihoods for ancestral inference. We apply MCMC to sample trees from the joint posterior distribution. Hence, measurement of uncertainty in 
our optimal tree accompanies tree construction. By contrast, other practical methods must first find an optimal tree, generate bootstrap samples from the data, and then reestimate the tree from each bootstrap sample to address uncertainty in their reconstruction.

This article is organized as follows. Section 2 opens with an introduction to the requisite terminology for tree representation. Section 3 presents a general stochastic model for the evolution of discrete molecular data. Section 4 articulates the Bayesian perspective and defines Metropolis-Hastings algorithms. Section 5 introduces a two-stage proposal distribution that randomly selects a canonical ordering of leaf labels, then acts on the superdiagonal of the corresponding cophenetic matrix. Section 6 describes restriction site data and posits a stochastic model for its evolution. MCMC is applied and key phylogenetic quantities are analyzed. Section 7 extends our method to nucleotide sequence data. Section 8 describes runs made with computer-simulated DNA sequence data from our model and reconstruction. Finally, we summarize the advantages of our approach and discuss further extensions to more complex models.

\section{Tree Terminology and Representation}

A phylogeny can be viewed abstractly as a rooted binary weighted tree. Mathematically, a tree is a connected graph $(V, E)$, with vertex set $V$ and edge set $E$, characterized by the absence of cycles. Vertices are classified as terminal nodes (also called leaves or tips) if they are connected through a single edge, and internal nodes otherwise, with $\mathcal{L}$ and $\mathcal{I}$ denoting the respective subsets. In rooted binary trees, each internal node has exactly three edges, with the exception of the root node $\rho$, which has only two edges. The placement of the root relative to the leaves determines the direction of time and hence ancestry. For each $v \in V \backslash\{\rho\}$, there is a unique parent node $\sigma(v)$, closer to $\rho$ and connected to $v$ by an edge in $E$.

\section{Sample Phylogeny on Seven Taxa}

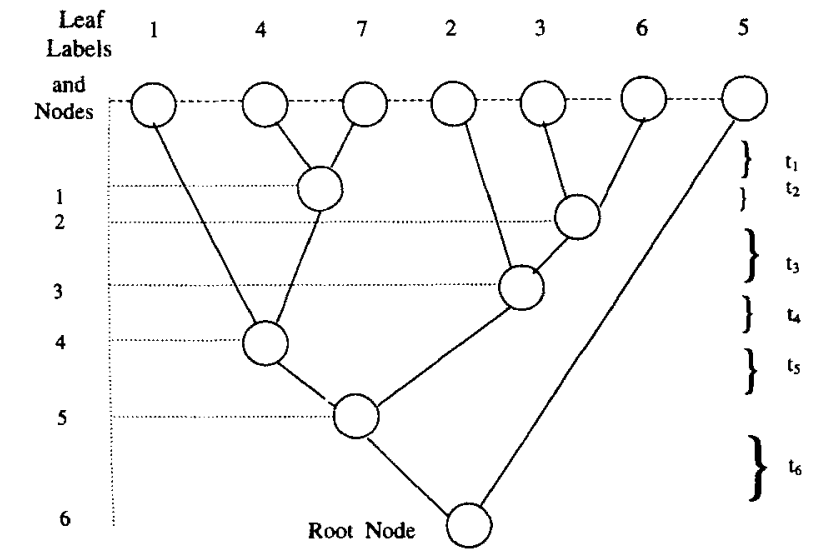

Coarescent Levels

and Coalescent Times

Figure 1. Labeled leaf nodes across the top identify present-day taxa. Internal nodes drawn below represent common ancestors, with connecting edges indicating lines of descent. $\{1,4,7\}$ and $\{2,3,6\}$ are examples of monophyletic groups (or clades), subsets of organisms whose most recent common ancestors have no other descendants among the considered organisms.
For expository reasons, we prefer to describe the branching pattern of a tree in terms of nodes coalescing or merging backward in time. Figure 1 illustrates the coalescence structure of a seven-taxa example.

The labeled shape of the tree, determined by which pairs of nodes coalesce, is called the tree topology and is equivalent to the graph $(V, E)$. The topology can be compactly summarized by using parentheses to indicate coalescences. For example, the topology in Figure 1 is $(((1(47))(2(36))) 5)$.

A weighted tree $\Psi$ is a tree in which each edge has an associated positive weight. The time separating a child from its parent is its edge weight, called its branch length. Branch lengths are the vertical distances between connected nodes in Figure 1. The ordering in which merges occur defines coalescent levels, and the corresponding temporal intervals between consecutive merges constitute coalescent times. Different orderings of coalescent levels within a particular topology generate distinct labeled histories, alternative characterizations of shape. We restrict attention to trees having contemporaneous tips, called dendograms. Such a tree $\Psi$ can be specified either by its topology and branch lengths or by its labeled history and coalescent times. The numbers of topologies and labeled histories grow rapidly with $n$, equal to $(2 n-3) \times(2 n-5) \ldots \times 1$ and $n ! \times(n-1) ! / 2^{n-1}$, respectively (e.g., Felsenstein, 1978).

For any weighted binary tree with labeled leaf nodes, the tree topology and branch lengths are determined by the within-tree distances between all pairs of leaf nodes (Lapointe and Legendre, 1992). Each permutation of the leaf labels generates a different $n \times n$ symmetric matrix of these distances. In a rooted tree in which all leaf nodes are equally distant from the root, such matrices are composed of at most $n$ distinct entries and are called cophenetic.

When a tree is displayed as in Figure 1, an arbitrary choice is made giving a left-right orientation to the pair of branches emanating from each internal node. This action imparts an order on the leaf nodes. The collection of $2^{n-1}$ orderings determined in this fashion is called the set of canonical orderings for a given tree. A cophenetic matrix with a canonical ordering has the desirable property that its superdiagonal (the diagonal of the submatrix formed by deleting the first column and $n$th row) contains each distinct nonzero cophenetic distance. We call such a cophenetic matrix canonical because its superdiagonal completely specifies the tree. One such matrix for the sample phylogeny of Figure 1 is realized in Table 1 . A convenient shorthand for the information sufficient to represent the tree is $\{\sigma, \mathbf{a}\}$, where $\sigma$ is a canonical ordering and a denotes the times to coalescence between adjacent label pairs in $\sigma$. Because times to coalescence are one-half the corresponding cophenetic distances, $\mathbf{a}$ is simply half the superdiagonal induced by $\sigma$ (for a detailed discussion of cophenetic matrices see Lapointe and Legendre, 1991).

\section{A Stochastic Model for Leaf Data}

Evolution has two components that can be modeled as stochastic processes: the branching created by speciation and extinction to form a phylogeny, and the propagation of characters along the branches of that phylogeny. We do not model the branching process stochastically, choosing instead to treat the phylogeny as a parameter in a model for the propagation 
Table 1

A canonical cophenetic matrix for the tree in Figure 1 , with canonical ordering $(5,7,4,1,2,6,3)$. For specificity, coalescent times $\mathbf{T}$ in Figure 1 are set at $(0.8,0.3,0.7,0.5,0.9,1.5)$, yielding a superdiagonal $(9.4,1.6,4.6,6.4,3.6,2.2)$. Redundant lower triangular entries are suppressed. The shorthand notation $(\sigma, \mathbf{a})$ becomes $\{(5,7,4,1,2,6,3),(4.7,0.8,2.3,3.2,1.8,1.1)\}$.

\begin{tabular}{cccccccc}
\hline \hline $\begin{array}{c}\text { Canonical ordering } \\
\text { of leaf labels }\end{array}$ & 5 & 7 & 4 & 1 & 2 & 6 & 3 \\
\hline 5 & 0 & 9.4 & 9.4 & 9.4 & 9.4 & 9.4 & 9.4 \\
7 & & 0 & 1.6 & 4.6 & 6.4 & 6.4 & 6.4 \\
4 & & & 0 & 4.6 & 6.4 & 6.4 & 6.4 \\
1 & & & & 0 & 6.4 & 6.4 & 6.4 \\
2 & & & & & 0 & 3.6 & 3.6 \\
6 & & & & & & 0 & 2.2 \\
3 & & & & & & & \\
\hline
\end{tabular}

of data along each lineage. We adopt standard Markov models for the second component (Goldman, 1990).

Typical character data on $n$ taxa can be arranged as an $n \times N$ matrix, where $N$ is the common number of sites, or positions, providing information from each taxa. We consider problems where elements of this matrix are discrete characters from a finite set $\mathcal{D}$ of size $d$. These data are viewed as a present-day snapshot of a realization of a stochastic process that has evolved along the branches of an unknown phylogeny $\Psi$. Modeling is reduced to a single site by assuming that evolution among sites is independent.

A stochastic model describes the joint distribution of $y=$ $\left\{y_{v}, v \in V=\mathcal{I} \cup \mathcal{L}\right\}$ of the historical record at $\mathcal{I}$ and the current status at $\mathcal{L}$ for a given site. The ancestral root state $y_{\rho}$ is assigned an initial distribution $\pi_{0}$ on $\mathcal{D}$. Conditionally on $y_{\rho}$, two continuous-time, $\mathcal{D}$-valued Markov processes emanate independently from the root $\rho$ along the corresponding branches of $\Psi$. As a given process reaches an internal node $v$, its value is recorded as $y_{v}$. Evolution continues by repeating this mechanism, with conditionally independent Markov processes emanating from every internal node $v \in \mathcal{I}$. Observable $y_{v}$ for $v \in \mathcal{L}$ are simply the end products of this evolution. Such a model for $y$ is specified by the phylogeny $\Psi$, the initial distribution $\pi_{0}$, and transition probabilities $p\left(y_{v} \mid y_{\sigma(v)}, t_{v}, \boldsymbol{\beta}\right)$. Here $\sigma(v)$ is the parent node of $v, t_{v}$ is the intervening branch length, and $\boldsymbol{\beta}$ is a parameter vector describing rates of change among states in the Markov process for a given branch. We consider two particular models in the examples in Sections 6 and 7 . The probability of the particular realization $y$ at a given site is

$$
\pi_{0}\left(y_{\rho}\right) \prod_{v \in V \backslash \rho} p\left(y_{v} \mid y_{\sigma(v)}, t_{v}, \boldsymbol{\beta}\right) .
$$

Both dependency between sites and site-specific $\beta$ 's can be incorporated into the model (e.g., Yang, 1996). Specification of an initial distribution $\pi_{0}$ should reflect the underlying biology or can be estimated from the observed frequencies in the data.

To calculate the likelihood function from leaf data at multiple sites, we must marginalize (1) over all values of the un- observed historical record $\left\{y_{v}, v \in \mathcal{I}\right\}$ for all sites. Straight summation is computationally prohibitive, requiring on the order of $N d^{n}$ calculations. The pruning method for likelihood evaluation requires on the order of $N d n$ computations because it takes advantage of the Markov property of the substitution model (e.g., Felsenstein, 1983). Pruning produces a collection of fragmentary likelihoods, starting from the leaves and working recursively to the root, for each site. For each leaf $v, L_{v}(i)=1\left[y_{v}=i\right]$ for state $i \in \mathcal{D}(1[\cdot]$ is the indicator function). At an internal node $v$, the conditional probability of descendant leaf data given $y_{v}=i$ is

$$
\begin{aligned}
L_{v}(i)= & \left(\sum_{j \in \mathcal{D}} L_{u}(j) p\left(j \mid i, t_{u}, \boldsymbol{\beta}\right)\right) \\
& \times\left(\sum_{m \in \mathcal{D}} L_{w}(m) p\left(m \mid i, t_{w}, \boldsymbol{\beta}\right)\right),
\end{aligned}
$$

where $\sigma(u)=\sigma(w)=v$. The likelihood function becomes a product across sites

$$
L(\Psi, \boldsymbol{\beta})=\prod_{k=1}^{N} \sum_{i \in \mathcal{D}} \pi_{0}(i) L_{\rho}^{k}(i),
$$

where the superscript denotes root fragmentary likelihoods at the $k$ th site.

\section{The Bayesian Perspective and Metropolis-Hastings Algorithms}

Bayesian analysis requires a prior distribution on the parameter space of the model. The prior should reflect the scientist's beliefs on how likely particular parameter values are before the data have been observed. The posterior distribution $\pi(\Psi)$ represents the uncertainty about the phylogeny in light of new evidence in the sequence data and is proportional to the likelihood times the prior distribution. In each example we consider, a uniform prior is placed on the finite set of labeled histories, and a flat prior density is assumed on a compact set of possible coalescent times as well as any propagation parameters from the stochastic model.

For large problems, Monte Carlo techniques might be the only effective way to integrate $\pi(\Psi)$ so as to obtain posterior inferences. One such method is the Metropolis-Hastings algorithm, in which a transition mechanism proposes a new tree $\Psi^{*}$ with density $Q\left(\Psi, \Psi^{*}\right)$, conditional on being at $\Psi$. We subject this draw to a randomized test, accepting it with probability

$$
\min \left(1, \frac{\pi\left(\Psi^{*}\right) Q\left(\Psi^{*}, \Psi\right)}{\pi(\Psi) Q\left(\Psi, \Psi^{*}\right)}\right)
$$

otherwise remaining at $\Psi$.

The Markov chain $\Psi_{1}, \Psi_{2}, \ldots$ so formed from an initial state $\Psi_{1}$ converges in distribution to $\pi(\Psi)$ when $Q$ is irreducible (e.g., Tierney, 1994). The important theoretical point is that for almost every realization of the chain,

$$
\lim _{K \rightarrow \infty} \frac{1}{K} \sum_{i=k_{0}+1}^{K+k_{0}} f\left(\Psi_{i}\right)=E_{\pi}[f(\Psi)],
$$


where $f$ is a function whose expectation is desired. For example, when $f$ is the indicator function of a particular topology, equation (4) means that the empirical relative frequency of that topology in the Markov chain converges to its corresponding (marginal) posterior probability. Credible sets, the Bayesian counterparts of confidence regions, are collections of topologies having high relative frequency in the chain. The topology with the highest relative frequency is our reported reconstruction.

Some initial sample points are discarded as burn-in $\left(k_{0}>0\right.$ in (4)) so as to reduce the bias in the Monte Carlo estimates (Besag and Green, 1993). We determine burn-in by inspecting time-series plots of the log posterior. A difficult problem with the implementation of MCMC is to know whether the $K$ used to approximate (4) is large enough. If $K$ is too small, then the Monte Carlo variance can overwhelm the signal (Geyer, 1992). Roughly speaking, a chain is said to mix well if it acts like an independent sample. Cowles and Carlin (1996) present a survey of convergence diagnostics commonly used to check for evidence of poor mixing. Though we emphasize the reproducibility of our results from random starts $\Psi_{1}$, we also analyze univariate statistics from single runs. Such diagnostic tools are helpful in ferreting out inefficient samplers but provide little insight into finding efficient ones. By considering distances in tree space, we have developed an algorithm that extends Bayesian analysis to relatively large problems.

\section{A Proposal Distribution for Trees}

We consider a two-stage proposal distribution. The first stage randomly selects a canonical representation $\{\sigma, \mathbf{a}\}$ for the current tree $\Psi$, whereas the second stage perturbs the components of a. In particular, the first stage $Q_{1}$ samples one of the $2^{n-1}$ canonical orderings of the current tree by independently flipping a fair coin at each internal node, thus selecting a particular superdiagonal $\left\{d_{i, i+1}: i=1, \ldots, n-1\right\}$ of a canonical cophenetic matrix having times to coalescence $\left\{a_{i}=d_{i, i+1} / 2\right\}$. The second stage $Q_{2}$ simultaneously and independently modifies the elements of a. Specifically, $a_{i}^{*}=\left|U_{i}\right|$, where $U_{i}$ is uniformly distributed on the interval $\left(a_{i}-\delta, a_{i}+\delta\right)$ for a tuning constant $\delta>0$. The tuning constant determines how far one can jump from the current tree and hence can be used to modulate the overall acceptance rate of the chain.

The reflection of uniform probability mass onto the positive line is an efficient way to obtain symmetric proposals near the boundary. Symmetry of each component update can be seen by inspecting the transition density

$$
Q_{2, i}(x, y)=\frac{1}{2 \delta}(1[|x-y|<\delta]+1[y<\delta-x])=Q_{2, i}(y, x),
$$

where $1[\cdot]$ is the indicator function and $x, y>0$. Regarding the composition $Q=\prod_{i} Q_{2, i} \circ Q_{1}$, the density at a possible proposed tree given the current tree equals

$$
\frac{1}{2^{n-1}} \prod_{i} Q_{2, i}\left(a_{i}, a_{i}^{*}\right)=\frac{1}{2^{n-1}} \prod_{i} Q_{2, i}\left(a_{i}^{*}, a_{i}\right) .
$$

The symmetry of $\mathrm{Q}$ simplifies (3), making this a Metropolis algorithm.

We illustrate the action of the second stage $Q_{2}=\prod_{i} Q_{2, i}$ in a second example, a six-leaved tree represented by $\{\sigma, \mathbf{a}\}=$ $(\{(4,6,3,2,1,5),(2.3,4.6,2.0,4.0,3.4)\}$. To recover the tree from the canonical cophenetic matrix, place the leaf nodes along a horizontal axis in their canonical order. From each interleaf midpoint, append an internal node below the axis a distance equal to the corresponding time to coalescence. Working from top to bottom, draw branches from each internal node to the nearest parentless nodes to the left and right (Figure 2). Newton, Mau, and Larget (1999) discuss this same proposal mechanism from a different perspective.

A candidate phylogeny proposed by $Q$ can differ from the current tree both in its labeled history and in its tree topology. For example, in Figure 2, when the perpendicular at $\{3,2\}$ is maximally increased while that at $\{4,6\}$ is simultaneously decreased, a new labeled history is proposed. Significantly, when a similar adjustment is made to the perpendiculars at $\{1,5\}$ and $\{2,1\}$, a different topology results, with $\{1\}$ coalescing with $\{2,3\}$ instead of $\{5\}$. Had the canonical ordering $(4,6,3,2,5,1)$ been chosen instead, the same realization by $Q_{2}$ would have coalesced $\{5\}$ with $\{2,3\}$.

We now establish irreducibility of $Q=Q_{2} \circ Q_{1}$. For a given permutation of labels, repeated applications of $Q_{2}$ clearly allow transit from any superdiagonal $\left\{2 a_{i}\right\}$ to any other superdiagonal. Hence, it is sufficient to show that $Q$ allows moves among any of the $n$ ! permutations of labels. A single application of $Q_{1}$ can move any label to the first position of the permutation. Repeated applications of $Q_{2}$ ensure that $2 a_{1}$ is the largest superdiagonal element, corresponding to a tree in which that first label is connected to the root by one long branch. Another application of $Q_{1}$ allows us to place any remaining label into the second position while fixing the first by choosing the current branch orientation at the root node. Successive applications of $Q_{2}$ yield a superdiagonal in which $a_{1}>a_{2}>a_{i}, i>2$, so that the second label coalesces directly into the penultimate internal node. By continuing the process we are able to move to any permutation. Hence, $Q$ is irreducible.

\section{An Example with Binary Data}

Sytsma and Gottlieb (1986) studied the evolutionary relationships among nine species of the genus Clarkia, plants indige-

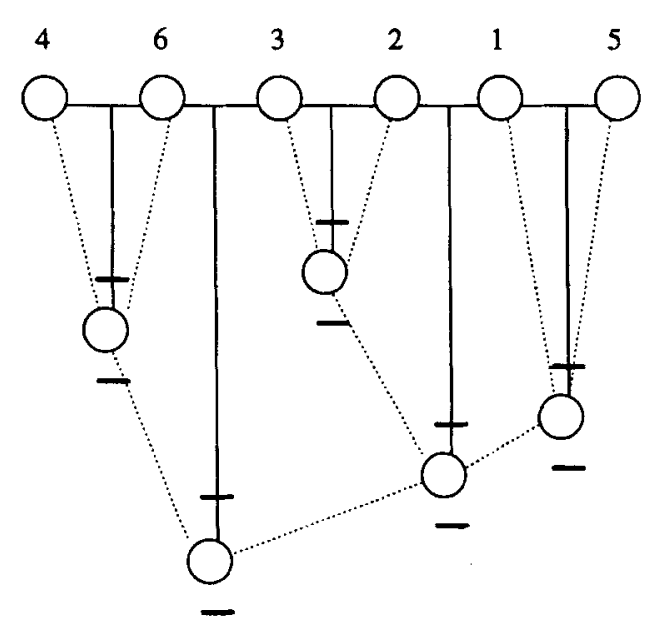

Figure 2. Candidate trees, attainable from the current tree, are characterized by intervals of size $2 \delta$, centered at the current internal nodes, that constrain the repositioning of the internal nodes. 
nous to California. These botanists extracted chloroplast DNA (cpDNA) from the leaves of a young plant of each species and exposed that cpDNA to 29 restriction enzymes. A restriction enzyme acts on DNA by physically cutting the molecule wherever it recognizes a particular base pattern. At each position on the genome where a cut occurs, a restriction site is said to be present. Sytsma and Gottlieb determined 609 positions where restriction sites occurred, 490 of which were noninformative (sites at every species). Informative data were translated into a $9 \times 119$ matrix of zeroes and ones, representing the absence or presence of a restriction site at mapped positions in the chloroplast genome for each species.

We entertain a simple stochastic model for the evolution of restriction sites; a two-state continuous-time Markov chain with infinitesimal rates $\lambda$ and $\mu$, representing the intensity of the instantaneous transition from 0 to 1 and 1 to 0 , respectively. The generator matrix is

$$
A=\left(\begin{array}{cc}
-\lambda & \lambda \\
\mu & -\mu
\end{array}\right) .
$$

The matrix $P(t)$ of transition probabilities through time $t$ satisfies the Chapman-Kolmogorov equation $P^{\prime}(t)=P(t) \cdot A$, with solution $P(t)=\exp (A t)$ having $(i, j)$ th entry

$$
p(j \mid i, t, r, \theta)=\frac{1}{1+r}\left(r^{1-j}+(-1)^{I\{i \neq j\}} r^{i} e^{-\theta t}\right),
$$

where $r=\mu / \lambda$ and $\theta=\mu+\lambda$ is the mutation rate.

We derive an initial distribution for restriction sites at the root from biological principles. A uniform distribution of nucleotide bases at the root and a single restriction enzyme with recognition sequence of length 6 suggest an initial distribution of $\pi_{0}^{*}(1)=(1 / 4)^{6}$ for the presence of a restriction site at each genomic site. Because recognition sequences differ from each other in at least two positions, the probability of a restriction site at each data site is about $\pi_{0}(1)=29 \pi_{0}^{*}(1)=0.00708$. Because a particular location in the genome is detected by the presence of a restriction site, only a minuscule fraction of the $170 \mathrm{~kb}$ (kilobases) enters the data. Following Felsenstein (1992), the likelihood (2) is conditioned on seeing at least one restriction site at each position

$$
L(\Psi, \boldsymbol{\beta}) /\left[1-p_{0}(\Psi, \boldsymbol{\beta})\right]^{N}
$$

where $p_{0}(\Psi, \boldsymbol{\beta})$ is the probability that $\left\{y_{v}=0, v \in \mathcal{L}\right\}$ at one site.

A complete Bayesian specification requires a prior on the propagation parameters $\boldsymbol{\beta}=(r, \theta)$. We place a uniform prior over $\left[1,4^{6}\right]$ on $r$, as constrained (somewhat liberally) by the biology, assuming a six-base cutter. The mutation rate $\theta$ is confounded with time, so the branch lengths are proportional to amount of evolution. Hence, we fix $\theta$ throughout our analysis. In the MCMC algorithm, we cycle between an update of the phylogeny $\Psi$ and an update of $r$. The proposal distribution for this second update is a uniform window centered at the current value.

We implemented the MCMC algorithm of Section 5 in Fortran 77 for this model and data set. From random starting trees, chains of length 250,000 were subsampled at a rate of 1 in 100 to reduce dependence in stored output, yielding 2500 phylogenies per run. Each run took approximately $20 \mathrm{~min}-$ utes on a Sparc 10 work station. Burn-in was less than 200 iterations, affecting only the first two stored samples. Convergence of the chain was inferred from the high degree of reproducibility of the posterior. In dozens of repeated runs from random starts, the realized relative frequencies deviate by at most $\pm 3 \%$, a measure of Monte Carlo error for samples of this size. Additional programming considerations for this data set are addressed in Mau and Newton (1997).

Figure 3 and Table 2 summarize our analysis of the Clarkia data. Topologies IV-VII have unrooted trees distinct from those that form the $99 \%$ credible region. Several subtopologies within the clade: $\{5,6,7,8,9\}$ are weakly supported by the data because of the placement of leaf $\{7\}$. Bootstrap analysis using parsimony attaches a weak $61 \%$ confidence coefficient to that branch in the optimal tree (Sytsma and Gottlieb, 1986 , p. 1257). By contrast, the posterior probability of an alternate attachment is a near negligible $0.4 \%$. The weakness in the rooting is reinforced when we simulate from a posterior that includes the outgroup species used by Sytsma and Gottlieb as a tenth taxa. That outgroup is attached beneath the $\{5,6,7,8,9\}$ clade with high posterior probability but not at the root.

Our assurances of convergence and adequate mixing of the chain are predicated on the verifiable reproducibility of the posterior. Visual tracking of a chain as it moves between the three predominant topologies provides further confirmation. That we are able to present fairly tight credible regions for the Clarkia data depends on both the nature and the amount of data but most dramatically on the number of taxa. In the next section, we increase the complexity of all three factors and provide additional diagnostics.

\section{A Nucleotide Sequence Example}

We have analyzed aligned protein coding mitochondrial DNA sequences obtained from 32 species of cichlid fishes (Kocher et al., 1995) using the HKY85 model of nucleotide substitution
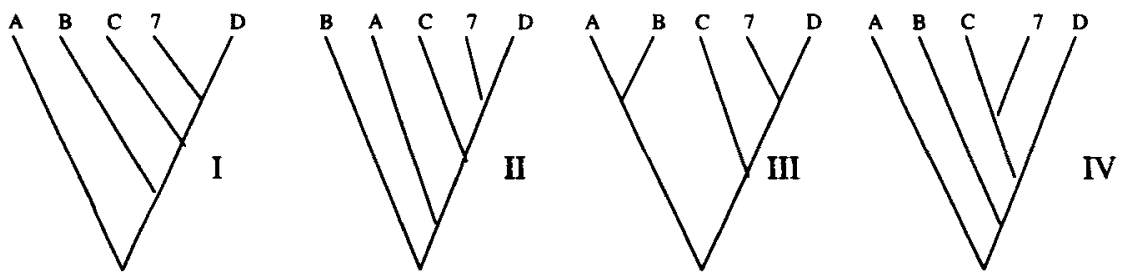

Figure 3. Shapes of the four most common topologies for the nine species of Clarkia, where $A=\{1,2\}, B=\{3,4\}, C=\{5,6\}$, and $D=\{8,9\}$ denote monophyletic pairs. The first three topologies differ only in the placement of the root. 
Table 2

Enumeration of the Clarkia topologies visited by the Markov chain, ranked by frequency of occurrence. Letters represent the clades defined in Figure 3. Topologies I through III constitute a $99 \%$ credible region.

\begin{tabular}{|c|c|c|c|c|c|}
\hline \multirow[b]{2}{*}{ Label } & \multirow{2}{*}{$\begin{array}{l}\text { Parenthetic representation } \\
\text { of the topology }\end{array}$} & \multicolumn{2}{|c|}{ Labeled histories } & \multicolumn{2}{|c|}{ Frequencies } \\
\hline & & Types & Counts & Relative & Cumulative \\
\hline 1 & $(\mathrm{~A}(\mathrm{~B}(\mathrm{C}(7 \mathrm{D}))))$ & 34 & 1621 & 0.649 & 0.649 \\
\hline II & $((\mathrm{A}(\mathrm{C}(7 \mathrm{D}))) \mathrm{B})$ & 28 & 447 & 0.179 & 0.828 \\
\hline III & $((\mathrm{AB})(\mathrm{C}(7 \mathrm{D})))$ & 28 & 419 & 0.168 & 0.996 \\
\hline IV & $(\mathrm{A}(\mathrm{B}((\mathrm{C} 7) \mathrm{D})))$ & 4 & 5 & 0.002 & 0.998 \\
\hline V & $((\mathrm{AB})((\mathrm{C} 7) \mathrm{D}))$ & 3 & 3 & 0.001 & 0.999 \\
\hline VI & $((\mathrm{A}((\mathrm{C} 7) \mathrm{D})) \mathrm{B})$ & 2 & 2 & 0.0008 & 0.9996 \\
\hline VII & $((\mathrm{AB})((\mathrm{CD}) 7))$ & 1 & 1 & 0.0004 & 1.0000 \\
\hline
\end{tabular}

Table 3

Tribal classification of 31 species of African cichlid fish. Taxa 1-5 form a flock from Lake Malawi. The remainder from Lake Tanganyika constitute a Tanganyikan flock. The Malawi, Ectodini, and Lamprologini tribes are represented by the letters $A, C$, and $D$, respectively. $B$ consists of $\{6,7,8,9\}$, a combination of most of Tropheini and one species of Limnochromini. $E=\{22,23,24,26,27\}$ and $F=\{28,29,30,31\}$ are convenient conglomerations (pseudoclades) of remaining tribes. Taxa $\{25\}$ is not grouped. Taxa $\{32\}$ is an outgroup from Central America.

\begin{tabular}{|c|c|c|c|}
\hline Label & Species name & Tribe & Clade \\
\hline 1 & Pseudotropheus zebra & Malawi & A \\
\hline 2 & Buccochromis lepturus & Malawi & A \\
\hline 3 & Champsochromis spilorhynchus & Malawi & $\mathrm{A}$ \\
\hline 4 & Lethrinops auritus & Malawi & A \\
\hline 5 & Rhamphochromis sp. & Malawi & A \\
\hline 6 & Lobochilotes labiatus & Tropheini & $\mathrm{B}$ \\
\hline 7 & Petrochromis orthognathus & Tropheini & $\mathrm{B}$ \\
\hline 8 & Gnathochromis pfefferi & Limnochromini & $\mathrm{B}$ \\
\hline 9 & Tropheus moorii & Tropheini & $\mathrm{B}$ \\
\hline 10 & Callochromis macrops & Ectodini & $\mathrm{C}$ \\
\hline 11 & Cardiopharynx schoutedeni & Ectodini & $\mathrm{C}$ \\
\hline 12 & Opthalmotilapia ventralis & Ectodini & $\mathrm{C}$ \\
\hline 13 & Xenotilapia flavipinnus & Ectodini & $\mathrm{C}$ \\
\hline 14 & Xenotilapia sima & Ectodini & $\mathrm{C}$ \\
\hline 15 & Chalinochromis popeleni & Lamprologini & $\mathrm{D}$ \\
\hline 16 & Julidochromis marlieri & Lamprologini & $\mathrm{D}$ \\
\hline 17 & Telmatochromis temporalis & Lamprologini & $\mathrm{D}$ \\
\hline 18 & Neolamprologus brichardi & Lamprologini & $\mathrm{D}$ \\
\hline 19 & Neolamprologus tetracanthus & Lamprologini & $\mathrm{D}$ \\
\hline 20 & Lamprologus callipterus & Lamprologini & $\mathrm{D}$ \\
\hline 21 & Lepidiolamprologus elongatus & Lamprologini & $\mathrm{D}$ \\
\hline 22 & Perissodus microlepis 1 & Perissodini & $\mathrm{E}$ \\
\hline 23 & Perissodus microlepis 2 & Perissodini & $\mathrm{E}$ \\
\hline 24 & Cyphotilapia frontosa & Tropheini & $\mathrm{E}$ \\
\hline 25 & Tanganicodus irsacae & Eretmodini & Unattached \\
\hline 26 & Limnochromis auritus & Limnochromini & $\mathrm{E}$ \\
\hline 27 & Paracyprichromis brieni & Cyprichromini & $\mathbf{E}$ \\
\hline 28 & Oreochromis niloticus & Tilapiini & $\mathrm{F}$ \\
\hline 29 & Tylochromis polylepis & Tylochromini & $\mathrm{F}$ \\
\hline 30 & Boulengerochromis microlepis & Tilapiini & $\mathrm{F}$ \\
\hline 31 & Bathybates sp. & Bathybatini & $\mathbf{F}$ \\
\hline 32 & Cichlasoma citrinellum & Central America & Outgroup \\
\hline
\end{tabular}




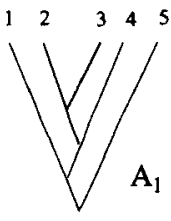

$((01((0203) 04)) 05)$

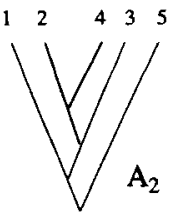

$((01((0204) 03)) 05)$

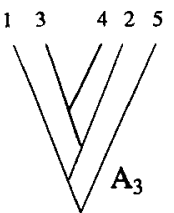

$((01(02(0304)) 05)$
Figure 4. Supported subtopological variation within the Malawi flock $A=\{1,2,3,4,5\}$. Variability is confined to coalescences involving taxa $\{2,3,4\}$. Marginal posterior probabilities are $0.776,0.183$, and 0.041 , respectively.

(Hasegawa, Kishino, and Yano, 1985). Table 3 shows some information about these species and their standard taxonomy. The HKY85 model is an example of the branching Markov substitution models discussed in Section 3. Four base composition parameters, $\pi_{A}, \pi_{C}, \pi_{G}$, and $\pi_{T}$ represent the long-run relative frequency of each nucleotide base in a single population, and these are used as the probability distribution of base values at the root of the phylogeny. The overall rate of substitutions is $\theta$. A parameter $\kappa$ characterizes the difference in substitution rates between transitions (changes between $A$ and $G$ or between $C$ and $T$ ) and transversions (any other change) (see Hasegawa et al., 1985, with $\alpha=\kappa \theta$ and $\beta=\theta$, for details). Each DNA sequence contains 1044 sites that can be partitioned into three blocks of sites according to codon position, and our analysis allowed different parameter values across blocks. Across all species, 567 of these sites are con- stant. Rather than entertain a full Bayesian analysis of this problem, we fixed parameters at estimated values and approximated the posterior distribution of the unknown phylogeny only (Appendix I discusses how we got parameter estimates).

There are more than $10^{40}$ topologies in this problem, as compared to $2 \times 10^{6}$ in the Clarkia example, thus presenting a significant challenge to model-based analysis. After extensive program testing, we can report with some confidence a Monte Carlo approximation to the posterior distribution. Our summary of most probable topologies was calculated by combining results from 10 independent realizations of the Metropolis chain. Each realization started at a phylogeny randomly sampled from the uniform prior distribution and proceeded for $1,100,000$ steps. The first $k_{0}=10^{5}$ steps were discarded as burn-in, and the remaining chain was subsampled every 200 steps to produce a stored sequence of 5000 phylogenies. The tuning parameter $\delta$ was selected adaptively during the initial stage of the run but attained a fixed post-burn-in value of 0.00098 in all 10 runs, equal to about $3 \%$ of the total height of a typical sampled tree. The acceptance rate of proposed trees was about $40 \%$ with this window. A C-language implementation using efficient likelihood evaluation and storage techniques took about 20 hours per realization on a Pentium 200 PC. Convergence diagnostics are described in Appendix II.

Summarizing the empirical distribution of sampled phylogenies presented a challenge. In the combined runs, we found that 34 topologies accounted for half the posterior probability. A $90 \%$ credible region contained nearly 600 different tree topologies. Evidently, much of this variation was caused by

Table 4

Most probable tree topologies from the combined runs for the cichlid fish data set. The posterior probabilities are averaged over the 10 separate runs. The standard deviation, minimum, and maximum of the computed posterior probabilities of the 10 separate runs are in the last three columns. Letters indicate monophyletic groups, and subscripts denote subtopologies within these clades. Subtopologies for the A clade are depicted in Figure 4.

\begin{tabular}{|c|c|c|c|c|c|c|}
\hline \multirow[b]{2}{*}{ Rank } & \multirow{2}{*}{$\begin{array}{c}\text { Parenthetic representation } \\
\text { of the topology }\end{array}$} & \multicolumn{2}{|c|}{ Posterior } & \multicolumn{3}{|c|}{ Variation among runs } \\
\hline & & Probability & Cumulative & $\mathrm{SD}$ & $\min$ & $\max$ \\
\hline 1 & $\left(\left(\left(\left(\left(\left(\left(A_{1} B_{1}\right) E_{1}\right) C_{1}\right) D_{1}\right) 25\right) F_{1}\right) 32\right)$ & 0.0714 & 0.0714 & 0.0062 & 0.0630 & 0.0844 \\
\hline 2 & $\left(\left(\left(\left(\left(\left(\left(A_{1} B_{1}\right) E_{1}\right) C_{1}\right) D_{1}\right) 25\right) F_{2}\right) 32\right)$ & 0.0567 & 0.1281 & 0.0080 & 0.0464 & 0.0708 \\
\hline 3 & $\left(\left(\left(\left(\left(\left(\left(A_{1} B_{1}\right) E_{1}\right) C_{1}\right) D_{2}\right) 25\right) F_{1}\right) 32\right)$ & 0.0342 & 0.1623 & 0.0088 & 0.0216 & 0.0458 \\
\hline 4 & $\left(\left(\left(\left(\left(\left(\left(A_{1} B_{1}\right) E_{1}\right) C_{1}\right) D_{2}\right) 25\right) F_{2}\right) 32\right)$ & 0.0293 & 0.1916 & 0.0062 & 0.0210 & 0.0422 \\
\hline 5 & $\left(\left(\left(\left(\left(\left(\left(A_{1} B_{2}\right) E_{1}\right) C_{1}\right) D_{1}\right) 25\right) F_{1}\right) 32\right)$ & 0.0271 & 0.2187 & 0.0064 & 0.0166 & 0.0366 \\
\hline 6 & $\left(\left(\left(\left(\left(\left(\left(A_{1} B_{1}\right) E_{2}\right) C_{1}\right) D_{1}\right) 25\right) F_{1}\right) 32\right)$ & 0.0238 & 0.2425 & 0.0024 & 0.0186 & 0.0264 \\
\hline 7 & $\left(\left(\left(\left(\left(\left(\left(A_{1} B_{2}\right) E_{1}\right) C_{1}\right) D_{1}\right) 25\right) F_{2}\right) 32\right)$ & 0.0215 & 0.2640 & 0.0057 & 0.0104 & 0.0278 \\
\hline 8 & $\left(\left(\left(\left(\left(\left(\left(A_{1} B_{1}\right) E_{2}\right) C_{1}\right) D_{1}\right) 25\right) F_{2}\right) 32\right)$ & 0.0200 & 0.2840 & 0.0041 & 0.0138 & 0.0274 \\
\hline 9 & $\left(\left(\left(\left(\left(\left(\left(A_{1} B_{1}\right) E_{1}\right) C_{1}\right) D_{1}\right) 25\right) F_{3}\right) 32\right)$ & 0.0188 & 0.3028 & 0.0028 & 0.0156 & 0.0232 \\
\hline 10 & $\left(\left(\left(\left(\left(\left(\left(A_{2} B_{1}\right) E_{1}\right) C_{1}\right) D_{1}\right) 25\right) F_{1}\right) 32\right)$ & 0.0171 & 0.3199 & 0.0022 & 0.0144 & 0.0210 \\
\hline 11 & $\left(\left(\left(\left(\left(\left(\left(A_{2} B_{1}\right) E_{1}\right) C_{1}\right) D_{1}\right) 25\right) F_{2}\right) 32\right)$ & 0.0139 & 0.3337 & 0.0021 & 0.0110 & 0.0176 \\
\hline 12 & $\left(\left(\left(\left(\left(\left(\left(A_{1} B_{1}\right) E_{1}\right) C_{2}\right) D_{1}\right) 25\right) F_{1}\right) 32\right)$ & 0.0124 & 0.3461 & 0.0041 & 0.0038 & 0.0170 \\
\hline 13 & $\left(\left(\left(\left(\left(\left(\left(A_{1} B_{1}\right) E_{2}\right) C_{1}\right) D_{2}\right) 25\right) F_{1}\right) 32\right)$ & 0.0102 & 0.3563 & 0.0033 & 0.0044 & 0.0152 \\
\hline 14 & 19 others & $\geq 0.0050$ & 0.4924 & & & \\
\hline 33 & 117 others & $\geq 0.0010$ & 0.7367 & & & \\
\hline 150 & 683 others & $\geq 0.0001$ & 0.9258 & & & \\
\hline \multirow{4}{*}{\multicolumn{2}{|c|}{$\begin{array}{l}B_{1}=(((0607) 09) 08) \\
B_{2}=(((0607) 08) 09) \\
C_{1}=((10(1112))(1314)) \\
C_{2}=(10((1112)(1314)))\end{array}$}} & $((15(1920))(($ & 7) 18$)) 21)$ & & \multirow{4}{*}{\multicolumn{2}{|c|}{$\begin{array}{l}F_{1}=((2829)(3031)) \\
F_{2}=(((2829) 30) 31) \\
F_{3}=(((2829) 31) 30)\end{array}$}} \\
\hline & & $(15(((1617) 1$ & $920))(21)$ & & & \\
\hline & & $((2223) 27)(2$ & & & & \\
\hline & & $((2223)(2426)$ & & & & \\
\hline
\end{tabular}


Biometrics, March 1999

\section{Table 5}

Marginal posterior probabilities for the top three subtopologies within each clade of cichlids. Subtopologies within clades are numbered in order of their posterior probability. Their combined posterior probabilities are summed. The final column is the probability that the constituent species are not monophyletic.

\begin{tabular}{cccccc}
\hline \hline Clade & $X_{1}$ & $X_{2}$ & $X_{3}$ & Combined probability & Probability not a clade \\
\hline A & 0.776 & 0.183 & 0.041 & 1.000 & 0 \\
B & 0.709 & 0.239 & 0.034 & 0.982 & 0 \\
C & 0.856 & 0.141 & 0.003 & 1.000 & 0 \\
D & 0.661 & 0.338 & 0.001 & 1.000 & 0.161 \\
E & 0.608 & 0.191 & 0.023 & 0.822 & 0.036 \\
F & 0.446 & $\mathbf{0 . 3 9 3}$ & 0.125 & 0.964 & \\
\hline
\end{tabular}

uncertainty in subtopological branching structure in the presence of fairly well supported monophyletic groups or clades. We have identified six such clades of between four and seven taxa, four of which appeared in every saved tree. Figure 4 shows three probable subtopologies for a clade of five species. Other subtopologies are defined at the bottom of Table 4 and simplify the presentation of topology uncertainty in that table.

Compelling evidence for the effectiveness of our MCMC algorithm is the reproducibility of probability estimates from independent realizations, especially because the starting positions arise from a uniform distribution. The last three columns in Table 4 quantify the variation from run to run in the posterior probability of individual topologies. For example, Monte Carlo standard error is less than $0.3 \%$ for the top 13 topologies.

Marginal probabilities are natural in Bayesian analysis and suggest further effective summaries of the posterior distribution. Table 5 shows the posterior distribution of subtopologies within clades. Clearly, the designation of clade is appropriate for $A, B, C, D$, whereas $F$ and especially $E$ are not necessarily monophyletic. Note that $B$ includes a member from the Limnochromini tribe, so it is somewhat surprising that this artificial clade is so unambiguously supported.

A second posterior summary is the distribution over clade trees, i.e., the uncertainty in how clades are connected to form the tree. Table 6 shows the most probable tree topologies, ignoring variability within clades. Notice that clades $A, B$, $C$, and $D$, the four clades with unanimous support in our samples, also are connected to one another unambiguously.

Finally, we compare our estimate with two phylogenies reconstructed using traditional methodologies. Kocher et al. (1995) use a distance matrix method (neighbor joining) on the third codon position to identify clades $A, B, C, D$, and $F$. The authors then apply maximum parsimony to four of those clades to obtain optimal subtopologies, rooting each with a taxon from an adjoining clade as an outgroup. We obtained a maximum likelihood estimate with the program Dnamlk from Phylip (version 3.572c), which, like our method, assumes a molecular clock that maintains a constant rate of mutation along each branch.

Because of our codon-position-specific model and different methodology, it is not surprising that our answer does not agree in whole with either reconstruction. There is a fair degree of similarity in the different solutions arrayed in Table 7 . Each estimate has clades $A, B, C, D$, and $F$ in common, reinforcing the current taxonomic scheme based on geographic proximity. The greatest disparity between estimates involves the attachment of taxa from clade $E$. Except for the control pair $\{22,23\}$, these species are dispersed throughout Lake Tanganyika. Interestingly, the three methods concur in placing the $B$ clade closer to the Malawi flock $A$, rather than

Table 6

Variation in the interclade coalescence of cichlids. Although $A$ and $B$ always merge together first, we continue to differentiate them to emphasize their disparate geographic origins. Uncertainty in the location of taxa in clade $E$ and taxon 25 cause almost all the variability at this level of summary.

\begin{tabular}{ccc}
\hline Clade trees & Posterior probability & Cumulative probability \\
\hline$(((((((A B) E) C) D) 25) F) 32)$ & 0.645 & 0.645 \\
$((((((A B) E) C)(D 25)) F) 32)$ & 0.102 & 0.747 \\
$((((((((A B)(2426))((2223) 27)) C) D) 25) F) 32)$ & 0.040 & 0.789 \\
$((((((((A B)((2223) 27))(2426)) C) D) 25) F) 32)$ & 0.033 & 0.821 \\
$(((((((A B) E) C) D) F) 25) 32)$ & 0.027 & 0.848 \\
$(((((((A B)((2223)(2426)) 27) C) D) 25) F) 32)$ & 0.024 & 0.872 \\
$(((((((A B) E) C) 25) D) F) 32)$ & 0.017 & 0.889 \\
$(((((((A B) E) C) D) 25)((2829) 30)) 31) 32)$ & 0.017 & 0.906 \\
$((((((((A B) 27)((2223)(2426))) C) D) 25) F) 32)$ & 0.015 & 0.921 \\
\hline
\end{tabular}


Table 7

Comparison of the Markov chain Monte Carlo estimate of the phylogeny for the cichlids with estimates using other methods. In the neighbor-joining solution, the use of [,] indicates how species in clade $E$ have been redistributed across clades. An additional subtopology is $B_{5}=((06(0708)) 09)$.

\begin{tabular}{ll}
\hline \hline \multicolumn{1}{c}{ Technique } & \multicolumn{1}{c}{ Topology } \\
\hline Neighbor-joining plus parsimony & $\left(\left(\left(\left[\left[\left(A_{2} B_{5}\right)\left[C_{2}(2223)\right]\right](2426)\right] 27\right)\left(D_{2} 25\right)\right) F_{3}\right)$ \\
Maximum likelihood & $\left(\left(\left(\left(\left(A_{2} B_{2}\right)((((2223) 24) 27) 26)\right) C_{2}\right)\left(D_{2} 25\right)\right) F_{2}\right)$ \\
Markov chain Monte Carlo & $\left(\left(\left(\left(\left(A_{1} B_{1}\right)(((2223) 27)(2426))\right) C_{1}\right)\left(D_{1} 25\right)\right) F_{1}\right)$ \\
\hline
\end{tabular}

to members from its own flock and in preferring a $B$ clade that admits a taxon from another tribe.

\section{Simulation Study}

Further support for the efficacy of our proposed algorithm came from a simulation study. We generated 10 synthetic data sets analogous to the cichlid data set. Each one had 32 aligned sequences of length 1044, separated into blocks of sites corresponding to the three codon positions, and each one was obtained using the simulation software Seq-Gen (version 1.04) from Rambaut and Grassly (1996). To mimic the complexity of the cichlid problem, we fixed the true phylogeny in this simulation equal to one sampled from the posterior in Section 7. In particular, the corresponding tree topology was the most probable one (Table 4). We also used the same substitution parameters as those obtained in the cichlid problem.

For each synthetic data set, we ran our MCMC algorithm twice, using the same burn-in, subsampling rate, and total chain length as before. Having two runs enables us to compare variation both within and between data sets and hence to gauge Monte Carlo error. The posterior distribution is fairly diffuse for all synthetic data sets, as it is in the cichlid problem. The posterior probability of the top 10 tree topologies ranges from 0.22 to 0.79 across the twenty runs with a mean of 0.49 , as compared to 0.32 in Table 4 . The number of tree topologies in a $90 \%$ credible set ranges from 20 up to 409 . This is smaller than the 600 or so topologies reported in the cichlid data analysis using all 10 runs but comparable to that from any single realization.

The variation between runs is small compared to the variation among synthetic data sets. Considering the probability assigned to the top 10 tree topologies, the absolute difference between runs was 0.01 on average. For any tree topology, the difference between runs in estimated posterior probability was consistently smaller than 0.021 .

The true tree topology was captured very well by the estimated posterior distributions: the modal tree topology was equal to the true one in 1 case, differed by a single branch placement in 8 cases, and differed by 2 branch placements for one synthetic data set. In 7 of the 10 cases, the true topology appeared in the top 10 . This is about what we expect when the average posterior mass assigned to the top 10 topologies is 0.49 . Overall, the posterior probability of the true tree topology ranged from 0.0076 to 0.3103 . Evidence is emerging that as we increase the number of sites of simulated data, more posterior mass is concentrated on the true tree topology.

\section{Discussion and Extensions}

The analysis of the Clarkia data set and restriction site model is straightforward, allowing us to introduce concepts and notation required in the more complex cichlid fish example. We therefore confine our remarks to the second, more challenging example.

Table 7 compares the estimates from the three methods but not the methods themselves. Both maximum parsimony and maximum likelihood have solutions that maximize objective functions determined by the data. By contrast, our method provides a distributional assessment in which pockets of high posterior probability are located as the chain traverses tree space. Our estimate of the phylogeny is a simple by-product of that assessment. We simulate a chain of trees where the longrun relative frequency of hitting any particular tree topology is proportional to its marginal posterior probability. For moderate number of taxa and a discriminating set of data, almost all tree topologies have essentially zero probability mass and as such are unseen in even extremely long chains. Nevertheless, reproducible samples from the posterior are generated efficiently. Other methods require bootstrapping to appraise the quality of their estimate. At best, a few hundred such phylogenies provide confidence coefficients on how well each node of the original estimate is supported by the data. Our method automatically provides posterior probabilities, as illustrated in Table 4 for the cichlids, from which confidence coefficients for a particular tree can be calculated if one so chooses.

For large numbers of taxa, other methods rely on incomplete heuristic searches and time-consuming rearrangements that do not guarantee to produce an absolute maximum. We claim a clear advantage in this regard because averaging over local maxima (tree islands in the phylogenetic context) goes to the very heart of MCMC technology. The likelihood surface for phylogeny can contain numerous local modes, depending on the number of taxa, the data, and the model. The proposal distribution we have developed appears to navigate reasonably well between modes in the two examples presented.

Our claim that we are accurately sampling from the posterior density of the cichlid fish example rests primarily on the close agreement in the estimates of posterior probabilities in different runs from widely dispersed random starting trees. We appreciate that researchers might not have the luxury of conducting multiple runs when run times approach a day in duration. To that end, time-series diagnostics and intraclade switching statistics from a single realization support our claim that the chain is mixing well given our level of subsampling. Notwithstanding, we echo the caveat of Cowles and Carlin 
(1996, p. 902) that no single convergence diagnostic is infallible and that making comparisons between a few parallel runs from disparate starting trees is necessary.

Our experience with the fish data indicates that stickiness can occur when one employs models that exhibit a significant lack of fit. For example, application of the standard HKY85 model equally to all sites generated chains with strongly autocorrelated $\log$ posteriors that did not mix within the $E$ pseudoclade. Modification of our method might be necessary to increase the rate of mixing within clades to successfully solve a wider range of data sets and models.

The technique described herein supposes a molecular clock. This constraint is a particular concern for the cichlid fish data, where significantly higher mutation rates have been observed among members of the Ectodini tribe (Kocher et al., 1995, p. 425). An alternate viewpoint considers additive trees in which leaves are not constrained to be contemporaneous and branch lengths are measured in units of evolution instead of time. We have implemented such a nonclock model by decomposing additive trees into dendograms and star components (see Lapointe and Legendre, 1992). The proposal distribution described in this paper is applied to the dendogram. A variant of $Q_{2}$, indexed by leaf labels, is applied simultaneously to the star component. The subsequent hybrid driver has been applied successfully to the cichlid data, and we plan to report the results elsewhere.

Our reported calculations have assumed rate constancy within blocks of sites, although models allowing more general rate variation are available that might further improve the fit (Yang, 1996). Independence between sites is a more difficult assumption to relax. Schöniger and von Haeseler (1994) look at protein coding regions where the first two codons are assumed correlated. General dependent models are described in Tavaré and Feng (1995). Fortunately, our method is independent of the particular form of the likelihood (or the prior). Provided that the likelihood is computable and the number of additional parameters in the model remains manageable, one should be able to substitute into the acceptance ratio and run the chain in a reasonable amount of time.

Since the original submission of this article, we have become aware of efforts of others to run Markov chains on the space of phylogenetic trees. Kuhner, Yamato, and Felsenstein (1997) use MCMC to sample genealogies to estimate the product of the effective population size and the mutation rate per site. Yang and Rannala (1997) and Li, Pearl, and Doss (1996) have proposed MCMC algorithms for Bayesian phylogenetic inference that differ greatly from the method we have developed. The global nature of the tree update, coupled with the existence of a tuning parameter that moderates the overall acceptance rate, distinguishes our approach.

\section{ACKNOWLEDGEMENTS}

The authors would like to thank Carter Denniston and David Baum for helpful discussions that provided the requisite biological background. Further thanks go to Tom Kurtz, Peter Donnelly, Simon Tavaré, Joe Felsenstein, Charlie Geyer, François Lapointe, Don Simon, and Brian Yandell for stimulating conversations during the evolution of this article. The suggestions of an anonymous reviewer regarding convergence diagnostics and computer-simulated data led to improvements in the final version.

\section{RÉSUMÉ}

Nous dérivons une chaîne de Markov pour échantillonner à partir de la distribution a posteriori pour un arbre phylogénétique conditionnellement à l'information de séquence provenant de l'ensemble correspondant des organismes, à un modèle stochastique pour ces données, et à une distribution a priori sur l'espace des arbres. Une transformation de l'arbre en une forme canonique de matrice cophénétique suggère une distribution simple et efficace pour sélectionner des arbres candidats proche de l'arbre courant dans la chaîne. Nous illustrons l'agorithme avec des données de site de restriction sur neuf espèces de plantes, puis nous l'étendons aux séquences d'ADN provenant de 32 espèces de poisson. L'algorithme fonctionne bien dans les deux exemples à partir d'arbres de départ aléatoires, en générant des estimations reproductibles et des ensembles plausibles pour le chemin d'évolution.

\section{REFERENCES}

Besag, J. and Green, P. J. (1993). Spatial statistics and Bayesian computation (with discussion). Journal of the Royal Statistical Society, Series B 55, 25-37.

Camin, J. H. and Sokal, R. R. (1965). A method for deducing branching sequences in phylogeny. Evolution 19, 311326.

Cowles, M. K. and Carlin, B. P. (1996). Markov chain Monte Carlo convergence diagnostics: A comparative review. Journal of the American Statistical Society 91, 883-904.

Evans, S. and Speed, T. (1993). Invariants of some probability models used in phylogenetic inference. Annals of Statistics 21, 355-377.

Felsenstein, J. (1978). The number of evolutionary trees. Systematic Zoology 27, 27-33.

Felsenstein, J. (1981). Evolutionary trees from DNA sequences: A maximum likelihood approach. Journal of Molecular Evolution 17, 368-376.

Felsenstein, J. (1983). Statistical inference of phylogenies. Journal of the Royal Statistical Society, Series A, 146, 246-272.

Felsenstein, J. (1985a). Confidence limits on phylogenies: An approach using the bootstrap. Evolution 39, 783-791.

Felsenstein, J. (1985b). Phylogenies and the comparative method. The American Naturalist 125, 1-15.

Felsenstein, J. (1988). Phylogenies from molecular sequences: Inference and reliability. Annual Review of Genetics 22, $1-65$.

Felsenstein, J. (1992). Phylogenies from restriction sites: A maximum likelihood approach. Evolution 46, 159-173.

Fitch, W. M. and Margoliash, E. (1967). Construction of phy. logenetic trees. Science 155, 279-284.

Geyer, C. J. (1992). Practical Markov chain Monte Carlo (with discussion). Statistical Science 7, 437-511.

Goldman, N. (1990). Maximum likelihood inference of phylogenetic trees, with special reference to a Poisson process model of DNA substitution and to parsimony analyses. Systematic Zoology 39, 345-361.

Goldman, N. (1993). Statistical tests of models of DNA substitution. Journal of Molecular Evolution 36, 182-198. 
Griffiths, R. C. and Tavaré, S. (1994a). Simulating probability distributions in the coalescent. Theoretical Population Biology 46, 131-159.

Griffiths, R. C. and Tavaré, S. ] (1994b). Ancestral inference in population genetics. Statistical Science 9, 307-319.

Hasegawa, M. and Kishino, H. (1989). Confidence limits on the maximum likelihood estimate of the hominoid tree from mitochondrial-DNA sequences. Evolution 43, 672677.

Hasegawa, M., Kishino, H., and Yano, T. (1985). Dating the human-ape splitting by a molecular clock of mitochondrial DNA. Journal of Molecular Evolution 22, 160-174.

Huelsenbeck, J. P. and Rannala, B. (1997). Phylogenetic methods come of age: Testing hypothesis in an evolutionary context. Science 276, 227-231.

Kocher, T. D., Conroy, J. A., McKaye, K. R., Stauffer, J. R., and Lockwood, S. F. (1995). Evolution of NADH dehydrogenase subunit 2 in East African cichlid fish. Molecular Phylogenetics and Evolution 4, 420-432.

Kuhner, M. K., Yamato, J., and Felsenstein, J. (1997). Applications of Metropolis-Hastings genealogy sampling. In Progress in Population Genetics and Human Evolution, Volume 87, P. Donnelly and S. Tavaré (eds), 183-192. New York: Springer-Verlag.

Lapointe, F.- J. and Legendre, P. (1991). The generation of random ultrametric matrices representing dendograms. Journal of Classification 8, 177-200.

Lapointe, F.- J. and Legendre, P. (1992). A statistical framework to test the consensus among additive trees (cladograms). Systematic Biology 41, 158-171.

Li, S., Pearl, D. K., and Doss, H. (1996). Phylogenetic tree construction using Markov chain Monte Carlo. Technical Report 583, Department of Statistics, Ohio State University, Columbus.

Mau, B. and Newton, M. A. (1997). Phylogenetic inference for binary data on dendograms using Markov chain Monte Carlo. Journal of Computational and Graphical Statistics 6, 122-131.

Navidi, W. C., Churchill, G. A., and von Haeseler, A. (1993). Phylogenetic inference: Linear invariants and maximum likelihood. Biometrics 49, 543-555.

Newton, M. A. (1996). Bootstrapping phylogenies: Large deviations and dispersion effects. Biometrika 83, 315-328.

Newton, M. A., Mau, B., and Larget, B. (1999). Markov chain Monte Carlo for the Bayesian analysis of evolutionary trees from aligned molecular sequences. In Proceedings of the AMS-IMS-SIAM Joint Summer Research Conference on Statistics and Molecular Biology, F. SeillierMoiseiwitsch, P. Donnelly, and M. Waterman (eds), Seattle, Washington, 1997, in press.

Rambaut, A. and Grassly, N. C. (1996). Seq-Gen: An application for the Monte Carlo simulation of DNA sequence evolution along phylogenetic trees. CABIOS 13, 235238.

Schöniger, M. and von Haeseler, A. (1994). A stochastic model for the evolution of autocorrelated DNA sequences. Molecular Phylogenetics and Evolution 3, 240-247.

Sinsheimer, J. S., Lake, J. A., and Little, R. J. A. (1996). Bayesian hypothesis testing of four-taxon topologies using molecular sequence data. Biometrics 52, 193-210.
Smouse, P. E. and Li, W.-H. (1989). Likelihood analysis of mitochondrial restriction-cleavage patterns for the humanchimpanzee-gorilla trichotomy. Evolution 43, 1162-1176.

Sokal, R. R. and Sneath, P. H. A. (1963). Numerical Taxonomy. San Francisco: Freeman.

Swofford, D. L., Olsen, G. J., Waddell, P. J., and Hillis, D. M. (1996). Phylogenetic inference. In Molecular Systematics, 2nd edition, D. M. Hillis, C. Moritz, and B. K. Mable (eds), 407-511. Sunderdland, Massachusetts: Sinauer Associates.

Sytsma, K. J. and Gottlieb, L. D. (1986). Chloroplast DNA evolution and phylogenetic relationships in Clarkia sect peripetasma (onagraceae). Evolution 40, 1248-1261.

Tavaré, S. and Feng, Y. (1995). Reconstructing phylogenetic trees when sites are dependent. DIMACS Technical Report $95-48,55-57$, Rutgers University, Piscataway, New Jersey.

Tierney, L. (1994). Markov chains for exploring posterior distributions (with discussion). Annals of Statistics 22, 1701-1762.

Yang, Z. (1996). Among-site rate variation and its impact on phylogenetic analysis. TREE 11, 367-371.

Yang, Z. and Rannala, B. (1997). Bayesian phylogenetic inference using DNA sequences: A Markov chain Monte Carlo method. Molecular Biology and Evolution 14, 717-724.

\section{Received July 1996. Revised November 1997. Accepted January 1998.}

\section{APpendix I}

\section{Estimation of Separate Parameters for Three HKY85 Models, One for Each Codon Position}

Exploratory data analysis of the cichlid DNA sequences by codon position shows striking differences in the percentage of variable sites $(31.5 \%, 14.9 \%$, and $90.5 \%$, respectively) and in base composition. We first estimated the values of $\pi$ separately for each codon position using observed base counts. To estimate different values of $\theta$, we ran three separate simulations using data from each position alone, updating $\kappa$ each cycle, and used the subsequent relative total tree heights to find values of $\theta_{1}=1.4, \theta_{2}=1.0$, and $\theta_{3}=8.3$. Finally, with these three values of $\theta$, we ran a chain with all the data allowing $\kappa$ to change separately for each position, estimating $\kappa_{1}=7.5, \kappa_{2}=2.5$, and $\kappa_{3}=10.75$. All subsequent runs were conducted with these parameter values fixed.

\section{APPENDIX II}

\section{Convergence Diagnostics for Cichlid Data Set}

The choice of chain length, subsampling rate, and burn-in parameters was affected by the limitations of computer speed and memory, but the analysis of output from test runs was also helpful. Figure 5 shows a time-series plot of the log likelihood of saved phylogenies from 1 of the 10 final runs. It is typical to see a dramatic increase in log likelihood during the burn-in period, with stabilization at what appears to be a stationary series after about $10^{4}$ basic steps. The autocorrelation function in Figure 6 indicates that trees separated by about 20 storage steps present approximately independent log likelihoods. 

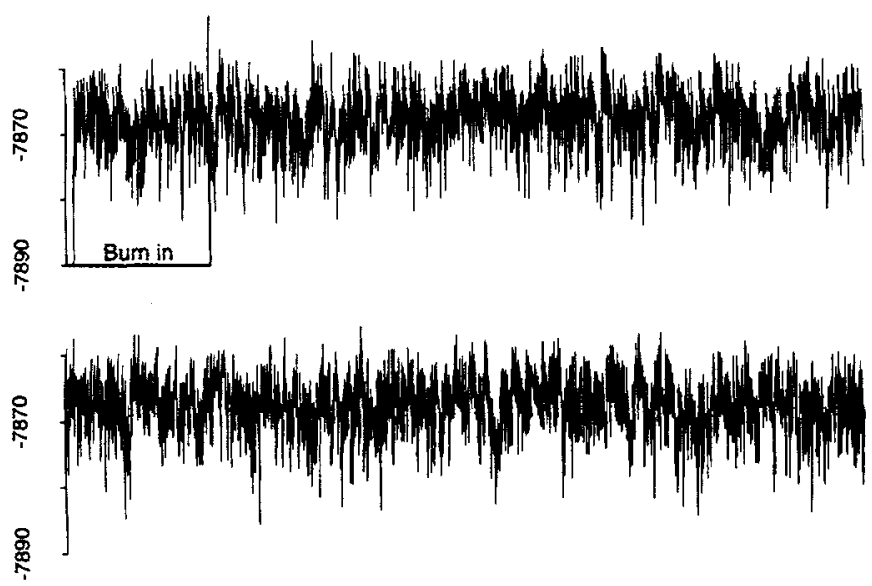

Figure 5. Time-series plot of the log posterior for 5500 subsampled trees of cichlids. The log posterior plateaus at a mean value of -7868 after 75 sample points, indicating that a burn-in of 500 is fairly conservative for this particular run. Lower panel shows the second half of our run.

An especially useful measure for assessing mixing is the frequency of topological changes within individual clades. Table 8 tabulates these switching counts from one run on the cichlid data. Ideally, the number of switches would be close to the expected number, assuming independent sampling. Indeed, Table 8 shows that the number of switches between the subtopologies within clade $A$ for one typical run agrees remarkably well with the expected counts, assuming independence shown here:

\begin{tabular}{lrrr}
\hline \hline & $A_{1}$ & $A_{2}$ & \multicolumn{1}{c}{$A_{3}$} \\
\hline$A_{1}$ & 3019 & 722 & 144 \\
$A_{2}$ & 722 & 173 & 34 \\
$A_{3}$ & 144 & 34 & 7 \\
\hline
\end{tabular}

The other clades do not mix as rapidly, with the number of observed switches only a small fraction of that expected assuming independence. The rate of mixing within these clades is sufficient, however, to give us reproducible results over separate runs.

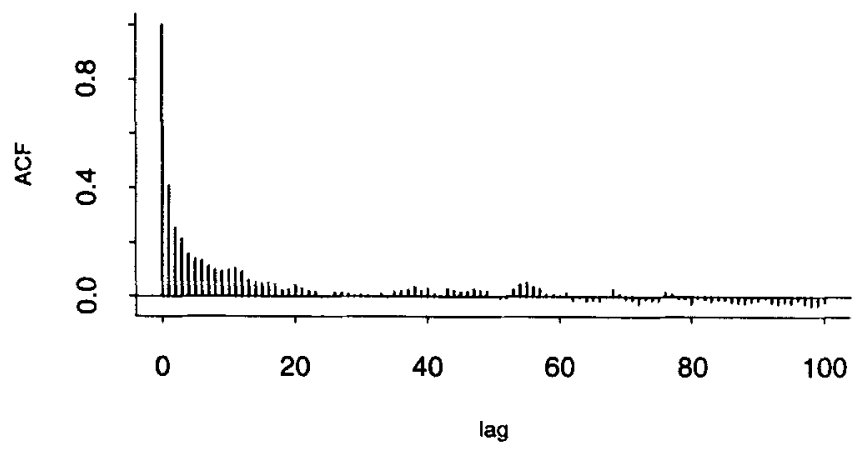

Figure 6. Autocorrelation function of the log posterior displayed in Figure 5. ACF drops below noise level at about 20 lags.

Table 8

Swapping between subtopologies for a single run on the cichlid data. Each row shows the distribution of the subtopologies that immediately follow a given subtopology in the saved sequences of trees. For example, subtopology $A_{2}$ followed $A_{1} 719$ times in the 5000 saved tree topologies. Topologies that appear only infrequently have been collapsed. A tree in which a clade does not appear is tabulated under--.

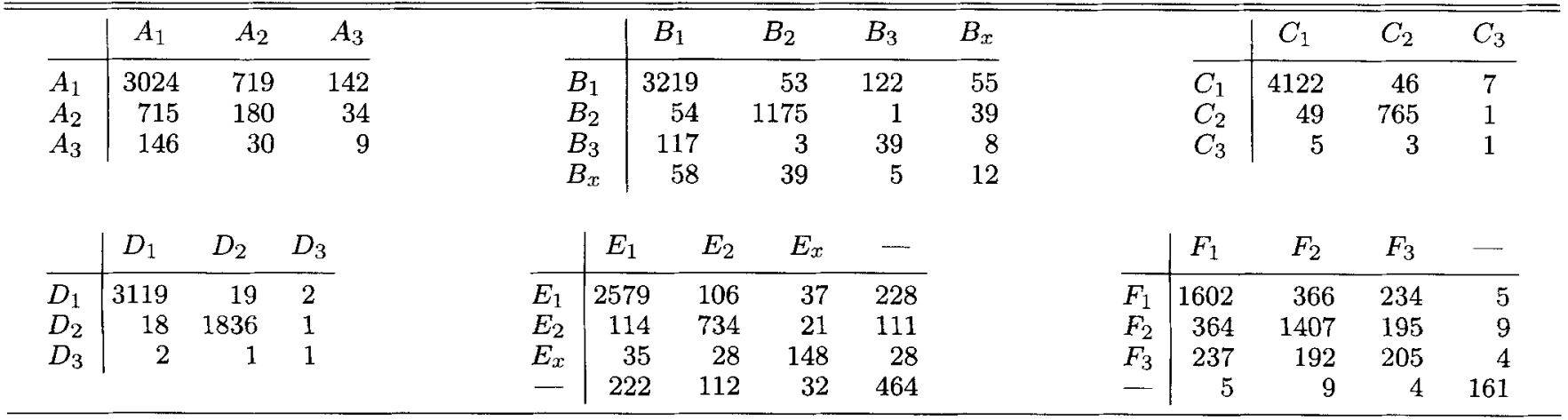

